\title{
Laparoscopic assisted total gastrectomy for gastric cancer - operative technique
}

\author{
Sebastian Smolarek, Abdelmonim Salih, Michal Kazanowski, Thomas N. Walsh \\ Royal College of Surgeons in Ireland, Department of Surgery, Connolly Hospital, Blanchardstown, Dublin, Ireland
}

Videosurgery Miniinv 2015; 10 (1): 133-137

DOI: $10.5114 /$ wiitm.2015.49092

\begin{abstract}
For many years, open gastrectomy with lymphadenectomy was the gold standard treatment for gastric cancer. In recent years, however, laparoscopic assisted total gastrectomy with associated D2 lymphadenectomy has gained in popularity. It has a similar oncological outcome to open resection, but has all of the added advantages of a laparoscopic procedure, such as early mobilisation, less postoperative pain and shorter hospital stay. This article describes the operative techniques, including key procedure steps, as well as a guide for using the new OrVil device for the laparoscopic creation of the oesophago-jejunal anastomosis. A video of a laparoscopic assisted total gastrectomy is presented.
\end{abstract}

Key words: OrVil, oesophago-jejunal anastomosis, Roux-en-Y.

\section{Introduction}

Gastric cancer is one of the most common malignancies worldwide, and surgery remains the gold standard treatment for locally advanced disease. The standard surgical procedure is a gastrectomy with D2 lymphadenectomy. For many years gastric surgery was performed through open resection, whilst in recent years laparoscopic distal gastrectomy has become more popular. This is especially evident in Asian countries. The available data suggest that laparoscopic partial gastrectomy has a similar oncological outcome to open resection and is in fact superior in terms of earlier mobilisation, less postoperative pain and earlier hospital discharge [1, 2]. There are unfortunately very limited data available in the literature for total laparoscopic gastrectomy (TLG) due to its technical difficulty. This difficulty is related to lymph node dissection, especially around the splenic hilum, and the creation of the oesophago-jejunal anastomosis. The introduction of the OrVil device to surgical practice seems to have been somewhat of a game changer with regards to the creation of the oesophago-jejunostomy, significantly reducing the overall technical difficulty of TLG [3].

\section{Case report}

This case report describes a laparoscopic assisted total gastrectomy in a 60-year-old man. The position of the patient is important during this procedure in order to obtain proper visualization and appropriate access for the laparoscopic instruments. The patient is placed on a bean bag in the Lloyd-Davis position with the table tilted $30-40^{\circ}$ head up. The surgeon stands between the patient's legs, with a camera operator on the patient's left while an assistant stands on the right. We use 5 ports in total for this operation.

The camera is introduced under direct vision using a visual port (Endopath Xcel Bladeless Trocar, Ethicon Endo-Surgery, Europe, $\mathrm{GmbH}$ ) to gain access

\footnotetext{
Address for correspondence

Sebastian Smolarek MD, RCSI Department of Surgery, Connolly Hospital, Blanchardstown, Dublin 15, Ireland,

e-mail: sebastian.smolarek79@gmail.com
} 
and create a pneumoperitoneum. This is inserted at three fourths of the distance between the xiphisternum and the umbilicus, 3 fingers to the left of the midline. Four further ports are then strategically placed in the abdomen under direct visualization. A $12 \mathrm{~mm}$ port is inserted into the left upper quadrant in the mid-clavicular line, just below the costal margin for the dissecting, clipping and stapling instruments. A $5 \mathrm{~mm}$ port is inserted lateral to it at the level of the Camera Port to facilitate retraction. Another $5 \mathrm{~mm}$ port is inserted in the right upper quadrant in the mid-clavicular line for the liver retractor, and finally a $5 \mathrm{~mm}$ port is introduced in the midline one third of the way between the xiphisternum and the umbilicus for the left handed dissecting instruments (Photo 1).

A $10 \mathrm{~mm}$ 30-degree lens is used. A SnowdenPencer articulating liver retractor is used to elevate the left lobe of the liver, introduced through a $5 \mathrm{~mm}$ port in the right upper quadrant. A $5 \mathrm{~mm}$ laparoscopic LigaSure system is used to divide the gastric vessels.

The dissection commences by dividing the omentum quite low on the greater curve of the stomach. This creates a "division of convenience" to facilitate mobilisation, because as soon as the stomach is resected the entire omentum is removed as a separate procedure. The lesser sac is opened and the omentum is dissected from the stomach upward towards the spleen. During the mobilization the left gastroepiploic artery is visualized, carefully dissected and ligated using an Endoclip. The clips are placed just above its origin from the splenic artery. As the dissection proceeds towards the fundus of the stomach, the short gastric arteries are divided using the

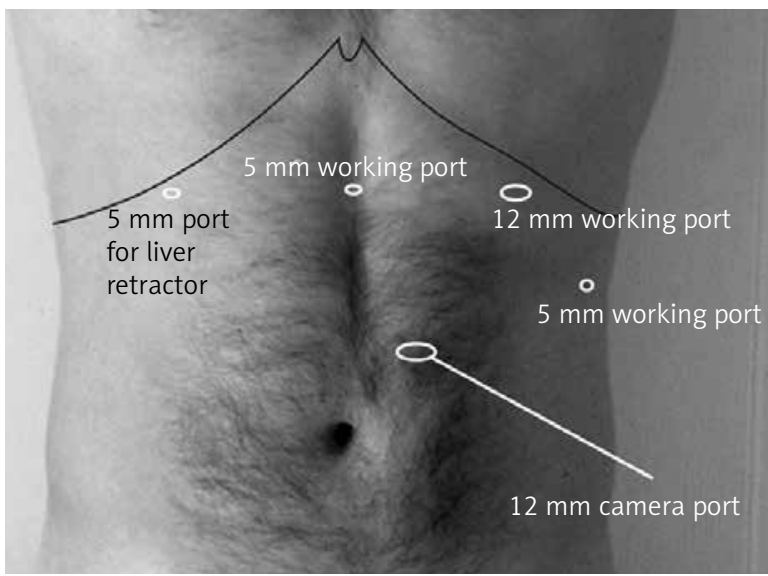

Photo 1. Port placement of laparoscopic gastrectomy
LigaSure. During this dissection, the lymph nodes from the splenic hilum are also removed.

Following the mobilisation of the upper part of the greater curve, dissection continues along the lower part of the greater curve towards the pylorus. The right gastroepiploic artery is then identified, carefully dissected and clipped using an Endoclip. The dissection then finishes at the pylorus.

After fully mobilising the greater curve, we focus on dividing the lesser omentum towards the oesophagus. The right diaphragmatic crus is then identified, and the dissection continues around the oesophagus to allow for full mobilisation of the abdominal oesophagus. When the oesophagus is fully mobilised, the dissection continues down toward the gastro-hepatic ligament. The right gastric artery is identified, dissected and ligated. Station 12 lymph nodes are gradually dissected en bloc with the stomach.

Once the lesser curve is fully mobilised, the first part of the duodenum is then divided using the Endo GA linear stapler (60 mm $3.5 \mathrm{~mm}$ ). This step allows the stomach to be lifted and accommodates further dissection, specifically the posterior stomach wall from the pancreas. During this dissection the splenic artery is fully visualised and the lymph nodes around the splenic artery are removed. Following this, the stomach is mobilised further towards the oesophagus. Continuing on, the left gastric artery is localised and a careful dissection of the lymph nodes around the left gastric artery and celiac trunk proceeds. After this dissection the left gastric artery is clipped, using Endoclips, close to its origin and is subsequently divided (Photo 2). At this stage the whole stomach is fully mobilised and devascularised. As a final step the abdominal oesophagus is divided using a linear Endo GA (60 mm $3.5 \mathrm{~mm}$ ) and the oesophageal remnants are allowed to retract back to the mediastinum. We then turn our attention to commence removing the omentum from the transverse colon using a LigaSure. On completion, the omentum and the stomach are placed into the bag and dropped into the pelvis for later retrieval.

Next, the ligament of Treitz is localised and the first loop of the small bowel is marked using a 3-0 Vicryl suture with length in the ends to allow for easier later identification and retrieval. We then progress to the next step of this hybrid operation, which involves a mini laparotomy through a $5 \mathrm{~cm}$ transverse incision in the left upper quadrant. A medium size Alexis wound protector is placed in the incision, 
through which the stomach and the omentum are removed in a bag. The first loop of the small bowel is again localised using the marking stitch and the loop is retracted through the mini-laparotomy incision. The jejunal segment with the best vascular arcade is selected for the creation of the Roux-en-Y limb. The arcade is visualised by trans-illumination using the laparoscopic light source outside the abdomen. The small bowel is divided using an Endo GA $(60 \mathrm{~mm}$ $3.5 \mathrm{~mm}$ ), and a Roux-en-Y end-to-side anastomosis is created using 4-0 Maxon sutures. After creating the Roux-en-Y limb, a marking stitch is placed on it, and this limb is subsequently returned to the abdominal cavity. The pneumoperitoneum is then re-established and the OrVil device is inserted through the mouth by the anaesthetist, to prepare the oesophagus for an anastomosis. The OrVil device (Covidien, Mansfield, MA, USA) is a DST Series EEA anvil assembly, with the anvil head secured in a tilted position. The assembly is mounted on a $90 \mathrm{~cm}$ long delivery tube, to which it is secured with a suture. This facilitates the passage of the anvil through the oral cavity, eliminating the need for a conventional hand-sewn purse-string closure (Photo 3). When used with the DST Series EEA XL 21 or $25 \mathrm{~mm}$ stapler, a circular, double staggered row of titanium staples is placed. In such cases we use a $21 \mathrm{~mm}$ anvil. The anvil is delivered down the oesophagus by the anaesthetist. When it impacts on the transverse staple line, the anaesthetist applies a steady pressure, and the surgeon cuts down on this using laparoscopic scissors (Photos 4 and 5). The delivery tube is then grasped and delivered through the $10 \mathrm{~mm}$ port. When the anvil head has impacted on the transverse staple line, the thread is divided and the delivery tube is disconnected.

The gloves from Alexis wound protector is removed and Roux-Y limb is actively retrieved through

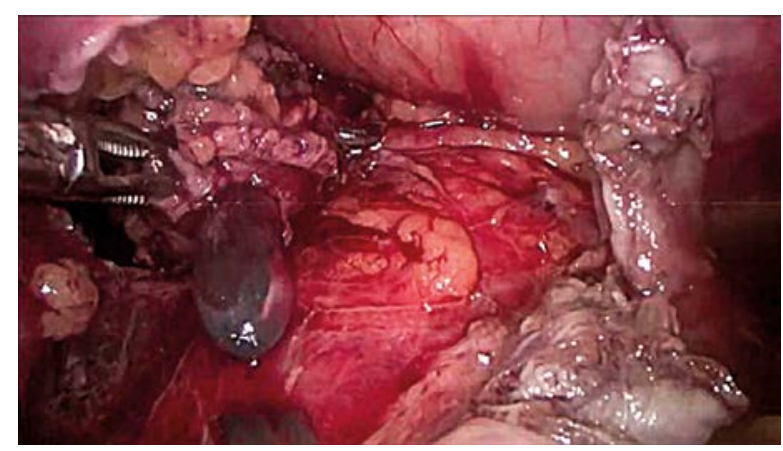

Photo 4. Delivery of OrVil anvil through oesophagus

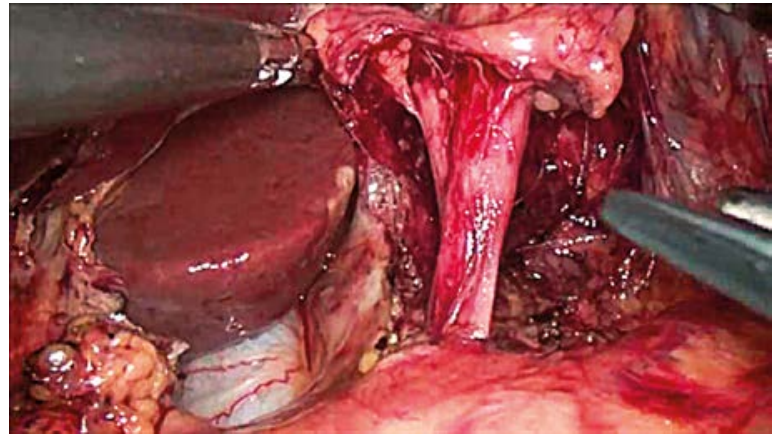

Photo 2. Left gastric artery prepared for apply-

ing the Endoclips

the mini-laparotomy. The CEA 21 circular stapling device is inserted into the middle finger of a size 8 glove to provide a seal over the Alexis wound protector as it is returned to the abdomen. It is then inserted into its long jejunal limb in preparation for the impending anastomosis.

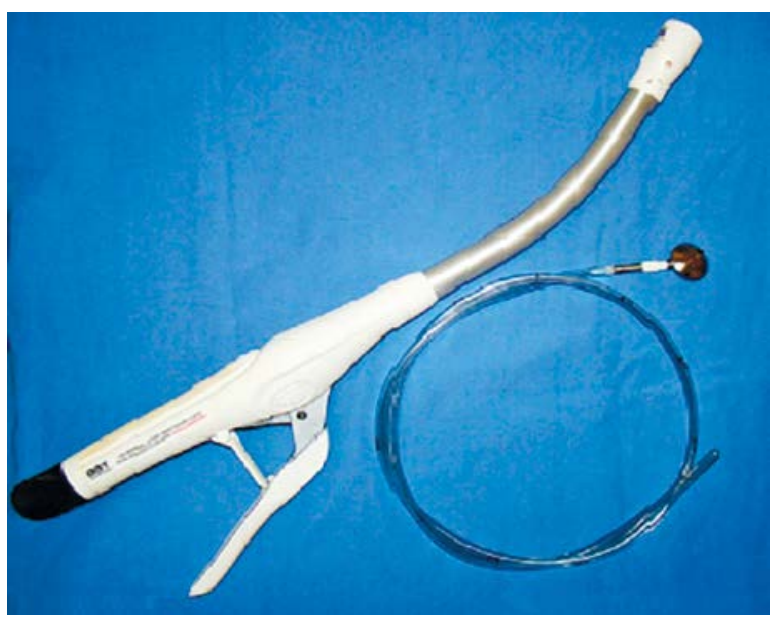

Photo 3. OrVil Device - DST EEA circular stapler device and trans-oral delivery system with anvil attached

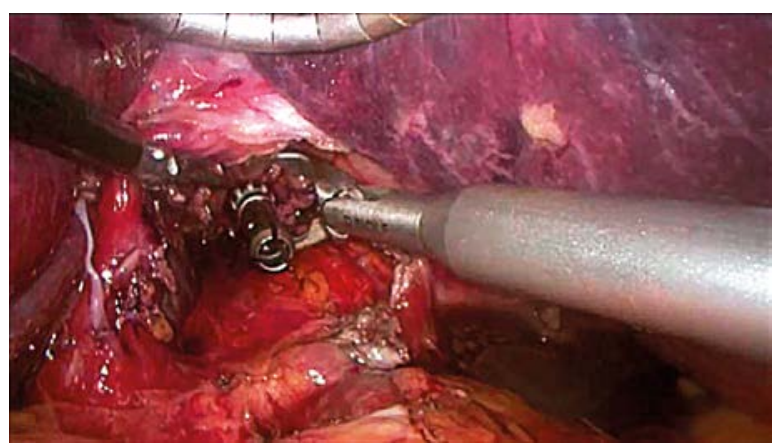

Photo 5. Preparing the anvil for anastomosis 


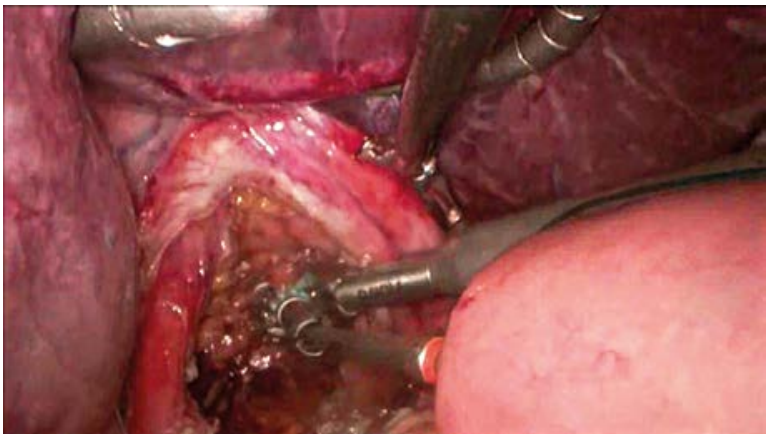

Photo 6. Connecting anvil with DST EEA stapler during creation of oesophago-jejuno anastomosis

We have a number of reservations about the functioning of the EEA device and have outlined our concerns with potential resolutions for these shortcomings in a separate publication [3].

The anastomosis is then created as was previously described [3]. One of our chief concerns here is that the spike of the EEA device is too sharp to be introduced back into the abdomen in an uncovered state. We have used a simple safety cover - the plastic sheath of a $25 \mathrm{G}$ needle (BD Microlance 3, Medisave (UK) Ltd) - to which we attach a long silk 0-0 suture to facilitate its later retrieval. A further relevant modification involves the use of Steristrips to secure the "knob" of the EEA device. The purpose of this is to ensure that the spike of the EEA gun withstands the pressure and does not collapse as it is being docked into the stem of the anvil, as significant manual force is usually required to complete this step accurately.

When the Roux limb is then mounted on the EEA stapler gun, it is secured using a 0-0 loop Maxon suture snagged over the spike plus a 4-0 Maxon suture to the edge of the limb. These steps are implemented to ensure that it does not slip as it is being returned into the abdomen. The stapler gun is then reintroduced into the abdomen with the surgical glove cuff secured on the Alexis wound retractor edge. This allows us to achieve a secure seal when the pneumoperitoneum is re-established.

When the camera is later reintroduced and the pneumoperitoneum has been restored, the EEA device is orientated and advances to create the anastomosis. The protective plastic "cap" is removed from the stapler spike; this is then docked and subsequently locked to the stem of the anvil (Photo 6). At this point the stapler is closed and the jejunal limb is carefully inspected to ensure that it is accurately positioned without twisting any of the mesentery. Finally the sta- pler is fired and the anastomosis is created. The EEA gun with its anvil is slowly and carefully withdrawn, first from the jejunal limb and then from the abdominal cavity. The donuts from the stapler are inspected, looking for any gaps, before sending for further histopathology examination. The blind end of the jejunum is shortened and closed using a linear Endo GA $60 \mathrm{~mm}$ (3.5 $\mathrm{mm}$ ). Prior to finishing, we secure the jejunal limb to the crus of the diaphragm with two non-absorbable sutures to eliminate any anastomotic tension.

Before closure, a mini Redi Vac drain is left in the left upper quadrant. The fascia of the mini laparotomy wound is then closed using two 0-1 loop PDS. The fascia in every port wound that is bigger than $5 \mathrm{~mm}$ is closed using a 0 Maxon suture. The skin is finally closed using 4-0 Maxon subcutaneous interrupted sutures.

The procedure lasts approximately $320 \mathrm{~min}$. The blood loss is approximately $50-80 \mathrm{ml}$. As a standard procedure after a total gastrectomy in our unit, we use total parenteral nutrition (TPN) until 7 days after the operation, and the patient is kept on nil by mouth for 6 days. We routinely perform a water soluble contrast swallow on day 5 to exclude any anastomotic leak prior to re-commencing an oral diet. After full enteral feeding is re-established on day 8 , the patient is discharged home.

\section{Discussion}

In recent years, we have seen a surge of interest in minimally invasive gastric surgery, with the result of laparoscopic distal gastrectomy becoming an important treatment option for distal gastric cancer. The use of laparoscopic or laparoscopic-assisted total gastrectomy is however somewhat limited. This is mainly due to the complexity and difficulty of creating the oesophago-jejunal anastomosis. Nevertheless, recent publications have reported better overall short-term outcomes in laparoscopic procedures compared to the open total gastrectomy. The laparoscopic approach offers less intraoperative blood loss, shorter postoperative hospital stays, less frequent occurrence of postoperative complications, shorter time to mobilization and shorter time to resuming normal dietary intake [4]. Ziqiang et al., in a long-term follow-up study, concluded that there was no significant difference in the 5-year survival rate between patients who underwent laparoscopic vs. open total gastrectomy [5]. 
The reported rates of laparoscopic oesophago-jejunal anastomotic leakage range between 1.8\% and $8 \%[6-8]$. In contrast to other authors $[9,10]$, who use a linear stapler for creating the oesophago-jejunal anastomosis, we used the OrVil device. We believe that using the OrVil device may actually decrease the anastomotic leakage rates, allow for a higher anastomosis in the mediastinum and also reduce the rate of positive resection margins. We feel that this device is most certainly a game changer in the world of gastro-oesophageal surgery.

In further comparison to other authors [5, 7], we do not use the mini laparotomy for resecting the cardia, nor for creating the gastro-jejunal anastomosis. We use the $5 \mathrm{~cm}$ incision, through which the specimen is removed, for the creation of the jejuno-jejunal anastomosis as an alternative approach. We believe that creating the Roux-en-Y $\operatorname{limb}$ as an open procedure provides a unique opportunity to properly identify the vascular arcade in the small bowel mesentery. This further allows for the creation of a lengthier Roux-en-Y limb, ensuring a very good blood supply and thus minimizing the risk of anastomotic leakage due to ischemia. The extended Rouxen-Y limb also allows for a tension-free oesophago-jejunal anastomosis, especially after the patient begins mobilization. Finally, it provides an excellent training opportunity for a hand-sewn anastomosis for younger trainees.

\section{Conclusions}

Laparoscopic assisted total gastrectomy is a technically demanding procedure which offers all of the benefits of laparoscopic surgery with the same oncological outcome as open surgery. The use of the OrVil device reduces the technical difficulties, shortening the learning curve, and may reduce postoperative anastomotic complications. Careful selection of the vascular arcade and creation of a longer proximal Roux-Y limb provide enough space for the creation of a well-vascularised and tension-free oesophago-jejunal anastomosis.

\section{Conflict of interest}

The authors declare no conflict of interest.

\section{References}

1. Huscher CG, Mingoli A, Sgarzini G, et al. Laparoscopic versus open subtotal gastrectomy for distal gastric cancer: five year result of a randomized prospective trial. Ann Surg 2005; 241: 232-7.

2. Lee SW, Nomura E, Bouras G, et al. Long-term oncologic outcomes from laparoscopic gastrectomy for gastric cancer: a single center experience of 601 consecutive resections. J Am Coll Surg 2010; 211: 33-40.

3. Salih A, Bass GA, D'Cruz Y, et al. Extending the reach of stapled anastomosis with a prepared OrVil ${ }^{T M}$ device in oesophageal and gastric cancer surgery. Surg Endosc 2014; 27 [Epub ahead of printing].

4. Li HT, Han XP, Su L, et al. Short-term efficacy of laparoscopyassisted vs open radical gastrectomy in gastric cancer. World J Gastrointest Surg 2014; 27: 59-64.

5. Ziqiang W, Feng Q, Zhimin C, et al. Comparison of laparoscopically assisted and open radical distal gastrectomy with extended lymphadenectomy for gastric cancer management. Surg Endosc 2006; 20: 1738-43.

6. Tsunoda S, Okabe H, Obama K, et al. Short-term outcomes of totally laparoscopic total gastrectomy: experience with the first consecutive 112 cases. World I Surg 2014; 38: 2662-7.

7. Lee JH, Ahn SH, Park DJ, et al. Laparoscopic total gastrectomy with D2 lymphadenectomy for advanced gastric cancer. World J Surg 2012; 36: 2394-9.

8. Jeong O, Ryu SY, Zhao XF, et al. Short term surgical outcomes and operative risks of laparoscopic total gastrectomy (LTG) for gastric carcinoma: experience at large-volume centrer. Surg Endosc 2012; 26: 3418-25.

9. Okabe H, Obama K, Tsunoda S, et al. Advantage of completely laparoscopic gastrectomy with linear stapled reconstruction: a long-term follow-up Study. Ann Surg 2014; 259: 109-16.

10. Pedziwiatr M, Matlok M, Kisialeuski M, et al. Enhanced recovery (ERAS) protocol in patients undergoing laparoscopic total gastrectomy. Videosurgery Miniinv 2014; 9: 252-7.

Received: 13.09.2014, accepted: 11.01.2015. 